\title{
Tomasz Placek
}

\section{ON BROUWER'S CRITICISM OF CLASSICAL LOGIC AND MATHEMATICS}

\begin{abstract}
The aim of this paper is to reconstruct Brouwer's justification for the intuitionistic revision of logic and mathematics. It is attempted to show that pivotal premisses of his argument are supplied by his philosophy. To this end, the basic tenets of his philosophical doctrine are discussed: the concepts of mind, causal attention, intuition of two-ity and his repudiation of realism. The restriction of intuitionistically allowable objects to spreads and species is traced back to Brouwer's concept of intuition that is a defining feature of his notion of mind. On the other hand, it is argued that his objections to some laws of classical logic result from the rejection of the rule of double negation elimination, which in turn follows from both, the claim that rules of logic should preserve evidence for assertions rather than truth, and too restrictive a concept of evidence.
\end{abstract}

\section{CONTENTS}

1. Introduction, p. 20

2. Mind, Time and Objects, p. 21

3. What mathematical objects are allowable?, p. 23

4. Replies to some possible objections, p. 24

5. What is logic?, p. 26

6. What is wrong with the principle of the excluded middle?, p. 27

7. Is there a way, from Brouwer's metaphysics to his concept of truth?, p. 29

8. Evidence, p. 31

9. Conclusions, p. 32

Received March 10, 1998 


\section{Introduction*}

Any revisionist program in logic and mathematics such as mathematical intuitionism should say why the recommended revision should be carried out. As it is easy to notice, the larger the gap between well-entrenched practises and proposed changes, the greater the demand for an answer to the above question. Obviously, a request to provide a justification for a proposed revision is of utmost importance in the case of a militant version of a revisionism, that is, a position that takes the proposed program to prescribe the only correct way of doing mathematics (or logic) and sees competing programs and the established practice as mistaken. Ideally, the militant revisionist should show three things. First, the established practise is faulty in some respect. Second, the fault is the result of more fundamental concepts or principles of classical mathematics or logic. Third, when the established practise is revised as suggested, the fault vanishes. On the other hand, the supporter of a policy of peaceful coexistence of various schools in the philosophy of mathematics (logic) needs to argue for a much weaker claim to the effect that the dictum 'de gustibus ...' does apply to mathematics.

Now, if we look from that perspective at the history of mathematical intuitionism, we will find out two rather strange facts. The first is that although intuitionism was generally considered a rather militant philosophy of mathematics, for the most of its history it has not had a justification or, at least, a proposed justification has not been known to a wider public. Although the founder of intuitionism, L. E. J. Brouwer, argued for his program in a number of papers, from 1907 untill the 1950's, few people have understood his philosophy and even fewer cared to discuss it. To the best of my knowledge, his philosophical position that offers arguments for inuitionism was not discussed, analyzed or questioned during his lifetime. His early followers either did not make mention of his philosophy or changed it considearbly, as if they did not value it. Lacking any other justification and fearing perhaps that Brouwer's metaphysical extravaganza could scare potential followers, from 1930's on A. Heyting advertised a peaceful co-existence policy. ${ }^{1}$

* The very first draft of this paper was presented at Wittgenstein Symposium in Kirchberg in 1992. The subsequent development of the paper owed much to Michael Dummett's comments. As to the final version, it was much improved thanks to John Vollrath's attempts to make clear both, its arguments and its English.

${ }^{1}$ This is readily seen in his 1956 book, Intuitionism. An Introduction. 
In more recent years support came, somehow surprisingly, from the philosophers' camp, thanks to Michael Dummett's semantical arguments. It is not, however, quite clear to me whether Dummett's position should be considered militant.

More troubling is the other peculiarity of intuitionism. Both Brouwer's and Dummett's justifications do not appeal to an alleged faulty practise of classical mathematics or logic alone, nor do they point to alleged logical or mathematical paradoxes resulting from an application of classical principles that intuitionism promises to cure. Instead, their rationale for replacing classical mathematics (logic) by intuitionistic one comes from the most general considerations of a metaphysical (Brouwer) or semantical (Dummett) nature. $^{2}$

I will concentrate here on Brouwer's ideas with an objective of reconstructing his justification of the intuitionistic revision in mathematics. It is my contention in this paper that Brouwer's line of argument rests on some epistemological and metaphysical premises that are explicit in his writings. To be more precise, the abilities that he assumes the knowing subject has, delineate what kind of knowledge is accessible to the human being, which in turn restricts mathematical objects that are (intuitionistically) allowable. Similarly, his criticism of the concept of mind-independent reality together with a rather peculiar views on the status of laws of logic motivates his rejection of some laws and principles of classical logic. What then, is Brouwer's epistemology?

\section{Mind, Time and Objects}

To present Brouwer's epistemological views one needs to sketch out a 'structure' of abilities, which, he assumes, the knowing subject has. Unfortunately, right at the beginning of this enterprise we encounter the most obscure notion of his philosophy, namely the notion of consciousness. The consciousness experiences sensations but in a way that seems to be more proper to dream-

\footnotetext{
${ }^{2}$ It is sometimes mistakenly believed that Brouwer's program arose as a reaction to paradoxes of logic and newly developed mathematical techniques that showed up in the second half of the nineteen century. As far as I know, however, the only place in which Brouwer discusses those issues is his doctoral dissertation (1907, pp. 80, 82, 83, 85). Rather shortly he analyses the paradox of the set of all sets, Russell's and Burali-Forti's paradoxes, the continuum problem and declares them to be linguistic phenomena arising only in a language that 'accompanies' mathematics, and not in mathematics itself. What's more, Brouwer does not even mention them in his late papers, in which he sketches the development of intuitionism.
} 
ing or being half-asleep than to being fully awake. As Brouwer put it, the consciousness 'seems to oscillate slowly, will-lessly and reversibly between stillness and sensation'. (1948, p. 480) Needless to say, this faculty is hardly suitable to such enterprises as mathematics, science or, in fact, any sort of rational thinking. It is rather a source of ultimate happiness and peace, a departure from which Brouwer considers an original sin of humans. Actually, there is such a departure in his system because some other faculty, which he calls temporal attention, enables a transition from the consciousness to a more developed stage of the knowing subject-namely to the mind. According to Brouwer, temporal attention is the splitting of every moment of life into something that is passing and something which is still 'now'. (1954, p. 522) Due to this, sensations become ordered according to whether they occurred earlier or later. One may say that the experience of inner time allows one to order meandering sensations. On the other hand, once the sensations are ordered, the separation of the flow of sensations and that which experiences them is born. Sensations appear to be independent of the subject and to originate from 'objective reality'. The separation brings about another effect, too. The consciousness, now fully separated from past and present sensations, becomes the mind. Its role maybe compared to that of a receiver: it receives an ordered flow of sensations.

Once the separation of the mind from its sensations is carried out, yet another faculty of the knowing subject comes into play. In the flow of sensations it identifies 'similar' sequences of sensations. That is, sequences of certain stability, such that their initial fragment is usually followed by the same series of sensations, are recognized to be similar. Such sequences are considered to be causal: the mind postulates an objective structure (a causal link or a causal chain) behind the sensations. For the role this faculty plays, it is called causal attention. Similarly to the construction of causal chains, the mind postulates an object behind a sequence of sensations whenever the members of the sequence are to a large extent alike. (1948, p. 480)

From the way objects and causal structures were introduced it should be clear that, according to Brouwer, they cannot belong to mind-independent reality. Their status is described by saying that they are correlative to the causal activity of the knowing subject. (1929, p. 418) However, once the object has been formed as a result of the causal attention of the subject, it looks as if the mind did not contribute to its formation. Hence, we can talk of two processes: the process of postulating an object (or a causal structure) and the process in which objects get the appearance of belonging to mind-independent reality. Both these processes are called objectification. 
Thus, one can say the concept of the mind plays three roles in Brouwer's epistemology: 1) it is the receiver of coming and passing sensations; 2) in the flow of sensations it identifies their various sequences; 3) it postulates a hidden reality behind the mosaic of sensations that in turn assumes the appearance of being mind-independent.

\section{What mathematical objects are allowable?}

Until now there has been no mention of those abilities of the knowing subject that have direct impact on Brouwer's concept of mathematics. As the name of that philosophy of mathematics suggests, the most important concept is the mathematical intuition which for the intuitionist is the sole source of mathematical knowledge. However, to get to the intuition one needs to remove all content from experienced sensations. ${ }^{3}$ Once the phenomenon of one sensation giving way to another is stripped of its sensory content, the 'empty form' of change is born, the form that is common to all changes experienced by the subject. Brouwer calls this empty form the 'basic intuition of two-ity' for he identifies change with the splitting of a moment of life into two parts, one of which passes by and gives way to the other. $(1952$, p. 510) As far as mathematics is concerned, the primary feature of the intuition consists in introducing and adding a new unit to the one already acquired by the mind. As the collection of added units is retained in the memory, this process leads to the construction of an arbitrary natural number. On the other hand, since the intuitionist allows not only for mathematical objects that can result from the application of rules of construction, but also believes that the rules themselves are legitimate mathematical objects, he or she has no objection to the notion of the infinitely proceeding sequence. The infinitely proceeding sequence is a special case of a more general concept called spread, which is roughly a combination of various infinitely proceeding sequences. Thus, one may say, the intuition furnishes the first sort of intuitionistic mathematical entities: spreads. The only other sort (called species) is an intuitionistic counterpart of the concept of set. However, in defining the species as a 'property supposable for mathematical entities previously acquired [... ]' (1954, p. 523) Brouwer assumes that species can only be introduced if the intuition allows one to create other mathematical objects. Consequently, it is the mathematical intuition that allows for the only two sorts of mathematical objects.

\footnotetext{
${ }^{3}$ The faculty at work here is called mathematical abstraction.
} 


\section{Replies to some possible objections}

The idea of founding exact knowledge on a concept of intuition has traditionally evoked criticism. It seems, however, that since Brouwer's concept of intuition diverges considerably from its traditional counterparts, mathematical intuitionism escapes that sort of criticism.

Firstly, for Brouwer intuition is not a source of certain mathematical knowledge, such that mistakes are excluded. The role of the intuition is limited to constructing mathematical objects. But in mathematical practice other faculties are also necessary, most notably memory. And, for Brouwer, it is the memory with its finitude that is responsible for mistakes. (1933, p. 443)

Secondly, it is even doubtful whether the intuition of two-ity can be correctly identified with a source of knowledge, if the latter means a faculty mediating between the mind and intuited objects. As was said before, the function of intuition consists solely in introducing mathematical objects to the mind. Now, if such a mental object is constructed, then it is immediately given to the mind. Hence, there is no need for a faculty that mediates between the mind and 'its' mathematical objects.

For similar reasons, Brouwer's concept of intuition can hardly be compared to an inner voice that helps you to guess the correct answers to mathematical puzzles. This is a concept of intuition that Wittgenstein occasionally attacks in his Philosophical Investigations. Intuition is identified there $(\S 213)$ with a faculty of guessing and choosing an interpretation of an initial sequence of some numerical series. That is, given such an initial fragment one can, in principle, interpret it in a number of ways, for example by finding various arithmetical expressions that describe the whole infinitely proceeding sequence. I assume Brouwer would totally agree that an ability should be postulated that accounts for the performance of such tasks. But why should this be identified with what he called 'intuition'?

Finally, the idea of founding mathematics on intuition of two-ity leads to the question as to how there can be an agreement between mathematical results obtained by different mathematicians, each of them following his or her 'own' intuition. A similar objection, but applied to counting, relies on asking whether the intuition can always bring a new unit. To remove these objections it is enough to notice an intimate link between the two basic concepts of Brouwer's philosophy: mind and intuition. The connection is as follows: the mind has to experience an ordered flow of sensations which simply means that any sensation is followed by another. If sensations are 
not ordered, then what receives them is not the mind. (This may be the consciousness, though). On the other hand, if you focus on the phenomenon of one sensation coming after another and strip off their sensuous content, you obtain the empty form of two-ity which is called the 'intuition'. Hence, the intuition is almost a defining feature of the mind: if something is the mind, then it has the ability called the 'mathematical intuition'. And similarly, there can be no talk of the intuition without the mind whose ability it is. Such an intimate link between mind and intuition makes Brouwer's epistemology almost immune to a criticism based on empirical data. Even if psychology were to discover a human being who does not experience an ordered flow of sensations and whose mathematical intuition sometimes stops bringing new units, this would not undermine Brouwer's philosophical views. The Brouwerian intuitionist would simply deny that such a human being has a mind, in the way this notion is understood by Brouwer. In the same vein, somebody who does not perceive a flow of sensations (because he is asleep or has taken drugs) cannot be said to have a mind. Similarly, if two mathematicians obtain different and unidentifiable answers to the same mathematical problem, then assuming the difference is not attributable to a flaw of a memory, it must result from a blank in functioning of the intuition. From what was said before, it follows that one or both mathematicians ceased to be (or have) the minds and this brought about a discrepancy in their results. ${ }^{4}$ This is a very speculative and ambiguous sort of reply. One may wonder, for instance, whether Brouwer's concepts of mind and knowing subject should be interpreted along Kantian lines or rather in a more subjectivist fashion. What is more, even if you feel satisfied with both the answer and its interpretation, you still face a more disturbing question, namely, what is the relation of the psychic subject to the epistemic subject, or how some abilities of a real mathematician are linked to the mind that Brouwer talks about.

\footnotetext{
${ }^{4}$ A short remark in the rejected part of Brouwer's doctoral dissertation (van Stigt 1979, p. 397) may suggest a yet another reply to the question of how the results of different mathematicians can be the same. While explaining his idea of mathematical 'objectification' of the world, Brouwer draws a distinction between the mathematical system and its emotional content. While properties of the former are independent of who constructed it, the latter changes from person to person. Obviously, it is the former that is the subject of mathematics. The only problem with the above reply is that there is no mention of that, or similar, distinction in Brouwer's later writings, when his epistemological ideas are getting more developed.
} 


\section{What is logic?}

Let us return to the question of how Brouwer's philosophical views influenced his conception of mathematics. I have already attempted to show how the assumed notion of intuition (or of mind) determines what mathematical objects are intuitionistically legitimate. But still more significant is another question, namely, what is his philosophical justification for the rejection of some classical laws of logic? The core of his argument seems to be the denial of common-sense realism together with his views on the nature of language.

Though linguistic phenomena belonged to Brouwer's field of interest, his views on the subject are hardly uniform. On the one hand, as one of the leaders of the significs movement, he was engaged in a project whose aim was firstly to describe and secondly to reform natural languages. The project relied on distinguishing various levels of natural language, each level presupposing another sort of extra-linguistic reality. (Brouwer 1919, p. 224, 229) Brouwer himself took the most extreme stance by insisting on 'cleaning' the language of harmful vocabulary. Words that could be used for demagogical purposes were to be removed from the language and replaced by other words capable of expressing humanist values. $(1937$, p. 452) On the other hand, he tried to account for the nature of linguistic phenomena in rather naive terms. The crucial idea that was supposed to explain language was that of one individual imposing his or her will on some other person with the help of articulated sounds and gestures. That notion served not only to explain how language originated in some distant past, but it was also intended as a partial account of more up-to-date phenomena of linguistic communications. (1929, p. 421)

Now, the problem is that for Brouwer laws of logic are intimately related to regularities observed in linguistic practise. If a sentence is asserted on the ground of appropriate evidence, let us call this assertion 'justified'. According to Brouwer, people observed that a justified assertion of a sentence of a certain type often allows one to assert justifiably a sentence of another sort. That is, they found that given evidence for the assertion of a sentence of the first type, they can also make the assertion of a sentence of some other sort because usually in such circumstances they could easily find the evidence for the assertion of the latter sentence. In other words, the transition from a sentence of one type to that of the other gets quite mechanical: the assertion of the latter sentence is made without the support of appropriate evidence, and there seems to be a guarantee that such evidence can be found whenever it is needed. As such transitions are rather regular, there must be some rules 
of transition, which Brouwer identifies with laws of logic. Since the rules are held to be empirical and, consequently, are assumed to depend on the characteristics of a language, he does not hesitate to demand that logical laws should be studied by the ethnographer rather than by the mathematician. (1907, p. 74)

\section{What is wrong with the principle of the excluded middle?}

Bearing in mind our problem of why some laws of classical logic should be rejected, the most important in the above story are two ideas. According to the first, logic is about rules that govern transitions between assertions of sentences. The second idea is the claim that the only correct transitions is that in which the evidence for assertion is preserved, that is, given the input sentence has a correct evidence for its assertion, the output sentence has it too. Remembering that rules of transition are identified with laws of logic, the claim means that a law of logic is valid only if it preserves evidence for assertions. Consequently, in the case of the transition from one sentence to the other that accords with a valid law of logic, one should be in a position to obtain the evidence for the latter sentence from the evidence for the former. Let us illustrate these ideas by considering an instance of the rule of double negation elimination.

Suppose we have evidence for the justified assertion of the sentence

It is not the case that there is no hairdresser store across the street.

Let us indulge in a little philosophical fiction and suppose that the evidence consists in the testimony of a perfectly reliable (that is, infallible) informer who claims that it is not the case that there is no hairdresser store across the street. Thus, the evidence is of a special sort: one cannot claim without further argument that it is also the evidence for the assertion that there $i s$ a hairdresser store across the street. Nevertheless, relying on the validity of the rule of double negation elimination, we assert the sentence

There is a hairdresser store across the street.

The evidence for the former sentence does not help to obtain the evidence for the latter. But in this case we can easily find this evidence. Just go out and look around! Provided our informer is perfectly reliable, and the number of stores on that street is finite, and we have good sight we will find a hairdresser store across the street. 
Thus, in this case, the assertion of the above sentence is justified. But validity of the rule seems to be a contingent matter: if we allow for undecided sentences (i.e., such that evidence is not available for both, the sentence and its negation), the rule in question will be no more valid. To illustrate this, let us focus our attention on Heyting's sigma example. (Heyting 1956, p. 17-18) To construct sigma, consider the possible occurrence of the series ' 123456789 ' in the decimal expansion of $\pi$. Now, if the last ' 9 ' of the series ' 123456789 ' does not occur in the $n$-th place of the expansion of $\pi$, put down ' 3 ' in the $n$-th place of the decimal representation of sigma. If such a ' 9 ' occurs in the $n$-th place of $\pi$, write ' 0 ' in the $n$-th place of the expansion of sigma and stop developing the expansion. Given this construction, we have evidence to assert the sentence

\section{It is not the case that sigma is not rational.}

However, this sort of evidence does not help us obtain the evidence for the sentence

\section{Sigma is rational.}

This is so, since for the intuitionist the only evidence for such a sentence must consist in calculating two integers $k$ and $l$ such that sigma equals $k / l$, we do not have the evidence for the assertion of the latter sentence and we may never have it. Hence, the rule of double negation elimination is not universally valid. Consequently, due to the rejection of this rule, a number of other classical laws of logic also stop being universally valid, most notably the principle of the excluded middle. Although, like in classical logic, the disjunction $p \vee q$ can be asserted on the ground of having either evidence for the assertion of $p$ or evidence for the assertion of $q$, it can not be asserted merely on the ground of the evidence for an assertion of $\neg \neg(p \vee q)$, and that makes a difference between the classical and the intuitionistic disjunction. It follows that there are cases in which the principle of the excluded middle does not hold intuitionistically: for any statement $p$ that has been neither proven nor disproved $p \vee \neg p$ does not hold.

A reader of Brouwer's papers may easily notice a discrepancy between our reconstruction of Brouwer's reasons for the rejection of the principle of the excluded middle and what he actually wrote on this subject. On the interpretation here defended, the pivotal part of the argument is the idea that the rule of double negation elimination is not universally valid. However, Brouwer's main concern in logic seems to be the principle of the excluded middle. What is more, he never writes about the rule of double negation 
elimination. Sometimes he discusses the principle of double negation elimination but usually such discussions follow after an argument against the principle of the excluded middle. So clearly, the order of issues in his papers diverges considerably from the order of importance suggested by our reconstruction. Hence, some words of explanation are needed. Brouwer's counter-examples to the principle of the excluded middle raise the question why it is claimed that a disjunction sentence $p \vee q$ holds only if there is evidence for asserting at least one of the disjuncts. Why may it not be asserted on the ground of having evidence for the assertion of $\neg \neg(p \vee q)$ ? Brouwer's counter-examples are hardly convincing because such a surreptitious change of assertion-conditions of the disjunction sentence makes a disjunction sentence in intuitionism mean something different from its classical counterpart. The change of assertion-condition for the disjunction sentence, however, is most easily explained by saying that the rule of double negation elimination is not assumed to be universally valid, which means that it cannot figure in the evidence for the justified assertions.

On this interpretation of Brouwer's argument, the rejection of some classical laws of logic results from the idea that rules of inference should preserve the 'availability of evidence' instead of preserving truth. Hence we need to say first what reasons Brouwer might have to urge such a replacement and second, explain the concept of evidence.

\section{Is there a way, from Brouwer's metaphysics to his concept of truth?}

Brouwer was very hostile to the concept of verification-transcendent truth. He often declared that 'there are no non-experienced truths'. (1948, p. 488) One may say that this attack on the concept of verification-transcendent truth was motivated by his repudiation of realism. Just assume, as Brouwer did, that any fact, mathematical or other, is a construction of the knowing subject carried out in accordance with some rules. So, you may say, there are no unconstructed facts. If you now assume that facts are expressed by true sentences, you will probably be inclined to repeat after Brouwer 'there are no non-experienced truths'.

As a matter of fact, however, I do not think the move that I am attributing to Brouwer, from the rejection of realism to the above notion of truth, is a coherent one. I brought it in because it points to a disturbing puzzle of Brouwer's intuitionism. Suppose there is an unknown fact, say, a mathematical problem $p$ that is not decided yet. Speaking in Brouwer's terms, 
if you try to solve it, you will follow the intuition of two-ity and attempt to arrive at one of two things: constructive proof that $p$ or a demonstration that from an assumption that there is such a proof, a contradiction follows. Now, the question is how rigid the rule of construction, that is, the intuition of two-ity, is. A plausible answer is that it is so rigid that any mathematical state of affairs is already determined, no matter whether anybody cares to construct it. If it is not so, then strangely the rule of construction leaves some indeterminacy as to whether something can or cannot be constructed. ${ }^{5}$

Well, maybe it was the concept of rule of construction, that led us astray. However, it is possible to restate the problem without invoking that concept! While discussing his counter-examples to the principle of the excluded middle, Brouwer often talks as if it were the existence of truly undecidable mathematical problems, that is the ground for objecting to this principle. He even equates the principle of the excluded middle with the claim that any problem can be decided, that is 'could either be proved or be reduced to absurdity'. (Brouwer 1981, p. 5). ${ }^{6}$ However, the concept of truly undecidable problem does not seem to have any meaning for the intuitionist. It cannot be constructed that $p$ means, due to the intuitionistic negation, $\neg p$. Similarly, It cannot be constructed that $\neg p$ means $\neg \neg p$. Thus, the assertion that some problem is truly undecidable must for the intuitionist boil down to a contradiction. It follows then that the intuitionist can only meaningfully talk about yet-undecided problems, which, abstracting from the fate of the human race, will either be proven or disproved in the future. This rather optimistic vision does not tell any reason why the principle of the excluded middle should be rejected.

At this point it is worthwhile to compare Brouwer's attack on the principle of the excluded middle with Łukasiewicz's rejection of bivalence. As it is reported in Łukasiewicz's "On Determinism", it was the indeterminate course of future events that made him give up bivalence and postulate three logical values. So, it was indeterminacy of future (or, anti-realism about future, in Dummett's terminology) that motivated the new logic. Although there are major differences between Eukasiewicz's three-valued logic and intuitionistic (two-valued) logic, it is exactly an appeal to some indeterminacy that is missing in Brouwer's line of argument. Even if we agree on Brouwer's understanding of the principle of the excluded middle, there is no answer for

\footnotetext{
${ }^{5}$ I owe this point to Professor John Vollrath.

${ }^{6}$ For a thorough analysis of how Brouwer understood the principle of the excluded middle, see (van Stigt, 1990), especially chapters 2.10 and 2.11 .
} 
why the intuition of two-ity does not determine every mathematical problem, such that it either can be constructed or cannot be constructed. Further more, Brouwer's understanding of the discussed principle together with his concept of negation, excludes a possibility of the sort of indeterminacy associated with the existence of truly undecidable problems. So, the remaining possibility is a sort of indeterminacy we encounter in indeterministic physics: the electron will take this or that slit. But here, I think, our intuitions will loudly rebel: speaking somehow metaphorically, the reason why a theorem is not yet decided is not because it has not yet 'decided' which way to go.

Thus, an important part of Brouwer's attacks on verification-transcendent truth and the principle of the excluded middle is missing. Even more, there are good reasons to believe that this line of argument cannot be improved. Thus, his ideas about truth does not follow from the rejection of realism, though the latter might motivate the former. With a remaining doubt as to why verification-transcendent truth should be replaced by a concept of evidence, let us examine the latter notion.

\section{Evidence}

As we saw in the above examples, the very crucial notion is that of evidence. For instance, in the case of Heyting's example, consider why the evidence for Sigma's being rational could only consist in calculating sigma and finding two integers, $k$ and $l$ such that sigma equals $k / l$. Why does a proof that it cannot be irrational not suffice? Contemporary intuitionists attempt to answer this question by arguing that there must be a hierarchy of proofs such that a sentence of lower logical complexity demands a proof of lower complexity. However, it is not clear from the epistemological point of view why such a ordering of proofs is needed. Equally importantly, the above idea has little credibility when applied to sentences of natural language and the evidence for their assertion. This is because the concepts of complexity of a sentence and complexity of evidence lose their precision in natural language discourse. One may speculate what Brouwer would answer to the objection that the intuitionistic concept of evidence is too restrictive. It seems to me that his reply would rest on a well-known distinction he made between mathematics and its accompanying language that serves to express its theorems, proofs and definitions. He believed that rules and principles of logic belong to this language and not to mathematics. Hence, mathematical evidence cannot rely on a rule of logic. $(1954$, p. 523) As the distinction is 
highly controversial, one should look for reasons for it and then certainly the search will again end among the basic tenets of Brouwer's philosophy: mathematical intuition, repudiation of realism, and the concept of the knowing subject.

\section{Conclusions}

I have attempted to show the connections between Brouwer's epistemology and the crucial ideas of his intuitionism. Firstly, the assumed concepts of mind and intuition have a direct impact on the decision as to what mathematical objects are intuitionistically allowable. Secondly, his repudiation of some classical laws of logic are traced back to his attack on mind-independent reality and to his views on the status of laws of logic.

In spite of the mentioned relations between Brouwer's philosophy and intuitionistic mathematics, his philosophy can hardly supply arguments in favor of replacing classical mathematics by intuitionism. And it is not the solipsism or psychologism allegedly inherent in Brouwer's philosophy that makes his arguments questionable. Quite to the contrary: the speculative character of his philosophy allows one to dismiss easily the objections mentioned above. The problem is a different one: there is simply little argumentation in Brouwer's philosophical papers. These works rather have the character of a revelation: Brouwer does not care to argue in favor of his concepts of mind, intuition and object. Similarly, he does not offer any grounds for identifying laws of logic with empirical generalizations. Further more, you can hardly find in his writings a rationale for the repudiation of realism. The arguments would not be necessary if his ideas were uncontroversial or at least plausible. But this is definitely not the case.

\section{References}

Brouwer, L. E. J.

CW Collected Works, vol. 1, ed. by A. Heyting, North Holland, 1975.

1907 "On the foundation of mathematics", in $C W$, p. 11-101.

1919 "Signifische Sprachforschung", in $C W$, p. 222-229.

1929 "Mathematik, Wissenschaft und Sprache", in $C W$, p. 417-428.

1933 "Volition, knowledge, language", in $C W$, p. 443-446.

1937 "Signific dialogues', in $C W$, p. 447-542. 
1948 "Consciousness, philosophy and mathematics", in $C W$, p. 480-494.

1952 "Historical background, principles and methods of intuitionism", in $C W$, p. $508-515$.

1954 "Points and spaces", in $C W$, p. 522-538.

1981 Brouwer's Cambridge Lectures on Intuitionism, ed. by D. van Dalen, Cambridge University Press, Cambridge.

Heyting, A.

1956 Intuitionism. An Introduction, North Holland, Amsterdam.

Łukasiewicz, J.

1954 "On determinism", in his Selected Works, North Holland, Amsterdam, 1970.

van Stigt, W. P.

1979 "The rejected parts of Brouwer's dissertation on the foundations of mathematics", Historia Mathematica 6 (1979), p. 385-404.

1990 Brouwer's Intuitionism, North Holland, Amsterdam.

Wittgenstein, L.

1958 Philosophical Investigation, Basil Blackwell, Oxford.

Tomasz PlaceK

Institute of Philosophy

Jagiellonian University

ul. Grodzka 52

31-044 Kraków, Poland

e-mail: uzplacek@cyf-kr.edu.pl 